\title{
Transition from spin-density-wave to layered antiferromagnetic state induced by hydrogen as a test for the origin of spin-density waves in chromium
}

\author{
V. M. Uzdin, ${ }^{1}$ H. Zabel, ${ }^{2}$ A. Remhof, ${ }^{3}$ and B. Hjörvarsson ${ }^{4}$ \\ ${ }^{1}$ Department of Chemistry, Saint-Petersburg State University, Universitetskij pr. 26, 198504 St. Petersburg, Russia \\ ${ }^{2}$ Department of Physics, Ruhr-University Bochum, 44780 Bochum, Germany \\ ${ }^{3}$ Empa, Swiss Federal Laboratories for Materials Testing and Research, Dübendorf, Switzerland \\ ${ }^{4}$ Department of Physics, Uppsala University, P.O. Box 530, 75121 Uppsala, Sweden \\ (Received 17 July 2009; revised manuscript received 14 September 2009; published 20 November 2009)
}

\begin{abstract}
Neutron scattering experiments of $\mathrm{Cr} / \mathrm{V}(001)$ superlattices are discussed, which show that the incommensurate spin-density-wave (SDW) in thick Cr layers becomes suppressed when the $\mathrm{V}$ spacer layers are loaded with hydrogen. The hydrogen loading triggers a transition from the incommensurate SDW state to a commensurate antiferromagnetic state. Model Hamiltonian calculations are presented, which show that this transition is not connected with the nesting property of the Cr Fermi surface. Instead, the transition is a manifestation of the antiferromagnetic ground state of $\mathrm{Cr}$, which is separated from the incommensurate SDW state by an energy barrier. Hydrogen is identified as an effective trigger for reducing the activation barrier, enabling the system to relax to the ground state.
\end{abstract}

DOI: 10.1103/PhysRevB.80.174418

PACS number(s): 75.30.Fv, 75.25.+z, 75.10.-b

\section{INTRODUCTION}

The nature of the incommensurate spin density waves (I$\mathrm{SDW}$ ) in $\mathrm{Cr}$ and in multilayers with $\mathrm{Cr}$ spacer layers is an open question despite the extensive experimental and theoretical efforts that have been undertaken for its elucidation. All recent $a b$ initio calculations show that the ground state for bulk $\mathrm{Cr}$ is antiferromagnetic ( $\mathrm{AF}$ ) or a commensurate spin-density-wave state (C-SDW), see Refs. 1 and 2 and references therein. $A b$ initio calculations reproduce the nesting property of I-SDWs, but the gain in energy due to the transition from paramagnetic (P) to I-SDW state is always less than from $\mathrm{P}$ to C-SDW state. Therefore, either all $a b$ initio calculations fail in the determination of the ground state for $\mathrm{Cr}$ or standard theories that presuppose that the nesting property of the Cr Fermi surface is the only reason for the I-SDW ground state should be revisited and eventually revised. Recently, we have put forward a theoretical approach based on the Periodic Anderson Model (PAM), which provides an explanation of the SDW state in $\mathrm{Cr}^{3}$ Despite the simplicity of this approach, which contains only three parameters for the description of each transition metal, in the case of $\mathrm{Cr}$, it reproduces most of the results obtained within the more sophisticated $a b$ initio approaches. In particular, the ground state of $\mathrm{Cr}$ corresponds to AF ordering, the magnetization profile for I-SDW solutions are very similar to those obtained within ab initio methods etc. Moreover, due to the use of analytical transformations instead of numerical integrations of the density-of-states during the self-consistency procedure, we were able to study the stability of the system relative to the local external magnetic field that so far cannot be done with $a b$ initio approaches. In our calculations, ${ }^{3}$ the transition to the I-SDW state was stimulated by the application of a local external and staggered magnetic field, which repeats the spatial distribution of the magnetization within the SDW. After switching off the magnetic field, the system remains in the SDW state if the SDW wavelength exceeds 40 ML. Furthermore, when the SDW period is longer than 51
MLs, the system cannot be transferred back to the AF state even after applying a staggered magnetic field on each site with changing in the field direction. Instead of a node annihilation, the nodes of the SDW shift and the system remains in the SDW state. Such a behavior can be considered as an excitation of nodes as quasiparticles on the $\mathrm{AF}$ ground state. These quasiparticles do not interact over large distances, but strongly attract each other and can even annihilate if the distance between them becomes less than 20-25 ML. Magnetic impurities and thermal excitations can easily destroy the nodes. This is considered to be the basic mechanism, which ultimately leads to a transition from I-SDW to AF in dilute $\mathrm{Cr}$ alloys with magnetic impurities and also explains the increase in the I-SDW period with increasing temperature.

The dynamical behavior of $\mathrm{Cr}$ magnetic moments at elevated temperature was analyzed by Aernout et al. ${ }^{4} \mathrm{Com}-$ parison of perturbed angular correlation measurements and neutron diffraction data reveals fluctuating magnetic moments in the $\mathrm{Cr}$ spacer of a Fe/Cr multilayer. They affirm that the observed spin fluctuations may be essential for the understanding of the SDW evolution with changes in temperature.

Fullerton et al. ${ }^{5}$ reported hysteretic SDW ordering in $\mathrm{Cr} / \mathrm{Cr}_{97,5} \mathrm{Mn}_{2,5}(001)$ multilayers. They observed a transition from AF to I-SDW order and discrete changes in the I-SDW period when cycling the temperature. Such behavior also can be associated with the dynamics of the I-SDW nodes. Thermal fluctuations can lead to a shift and diffusion of the nodes, and even to their annihilation due to the interaction with other nodes or with interfaces. In layered structures, the movement of the nodes in the direction perpendicular to the layers is restricted, and if the distance between nodes is of the same order as the radius of their interaction, they will not be able to move across interfaces but will only annihilate with increasing temperature. Change in the number of nodes leads to a discrete change in the SDW wavelength, and these transitions show hysteretic behavior because of the relative 
stability of the nodes. If the distance between nodes is larger than the radius of their interaction, thermal fluctuations lead to a translational movement of the nodes without change in their separation. In this case, the excitations of the nodes are harder to detect experimentally and hysteretic effects are hidden, as observed in neutron scattering experiment. ${ }^{5}$

For an experimental verification of the suggested SDW formation, it is important to find a way to continuously tune the SDW state. This can be done, for instance, via interface alloying, via adsorption of hydrogen at free $\mathrm{Cr}$ surfaces, or via doping $\mathrm{Cr}$ interfaces in layered structures with hydrogen. Utilization of finite-size effects in thin $\mathrm{Cr}$ films provides an additional possibility to test the SDW state. ${ }^{6}$

Mibu and Shinjo ${ }^{7}$ demonstrated that the magnetic structure of SDWs in Cr can be modified by a periodic insertion of nonmagnetic monatomic layers, using neutron scattering and Mössbauer spectroscopy with ${ }^{119} \mathrm{Sn}$ probe layers as a probe. Nonmagnetic $\mathrm{Sn}, \mathrm{Ag}$, and $\mathrm{Au}$ layers tend to increase the $\mathrm{Cr}$ magnetic moments at the interface, whereas $\mathrm{V}$ atoms suppress the magnetic moment of neighboring $\mathrm{Cr}$ atoms. This leads to a pinning of the SDW antinodes by $\mathrm{Sn}, \mathrm{Ag}$, and $\mathrm{Au}$ layers and of the nodes by $\mathrm{V}$ layers. Thus, in such superlattices, the SDW wavelength is no longer determined by the intrinsic $\mathrm{Cr}$ properties, but by the superlattice period and the boundary conditions. The I-SDW state is transformed to the AF state due to thermal excitations, which destroy the SDW nodes with increasing temperature. Moreover, the AF state is observed in superlattices with relatively short periods ( $\leq 40 \AA)$, because I-SDWs with short modulation period are not stable with respect to a transition to the AF state. ${ }^{3}$

Studies of SDWs in $\mathrm{Cr} / \mathrm{V}$ multilayers are of particular interest because of the possibility to modify the magnetic structure via hydrogen absorption in the V layers. However, it should be kept in mind that not only hydrogen but also the interface mixing has a dramatic effect on the SDW state in $\mathrm{Cr} / \mathrm{V}$ superlattices. Almokhtar et al. ${ }^{8}$ detected a sizable magnetic moment already at a distance of $5 \AA$ from the interface in $[\mathrm{V}(10 \AA) / \mathrm{Cr}(80 \AA)](001)$ superlattices. In contrast, Kravtsov et al. ${ }^{9}$ reported a complete suppression of $\mathrm{Cr}$ magnetism in $\mathrm{V} / \mathrm{Cr}(001)$ superlattices for Cr layers that are less than $90 \AA$ thick. Fritzsche et al. ${ }^{10}$ found in (110) oriented $\mathrm{V}(50 \AA) / \mathrm{Cr}(300 \AA)$ superlattices a complete suppression of the I-SDW, instead they observe an AF state down to the temperature of $2 \mathrm{~K}$. The authors explained this suppression by the elastic strain effects due to the lattice mismatch at the interface. In contrast, Rotenberg et al. ${ }^{11}$ observed an I-SDW state in $\mathrm{Cr}(110)$ films grown on $\mathrm{W}(110)$ substrates already at thicknesses below $80 \AA$ despite a $9 \% \mathrm{Cr}-\mathrm{W}$ lattice mismatch. On the other hand, Jiko et al. ${ }^{12}$ detected in $\mathrm{Cr}(110) / \mathrm{Sn}$ multilayers a coexistence of $\mathrm{AF}$ and I-SDW states for a $\mathrm{Cr}$ thickness of $160 \AA$, but for a thickness $80 \AA$, only AF ordering was found.

Such inconsistencies in experimental results reported by different groups is not surprising, because already $3.8 \%$ of $\mathrm{V}$ in dilute $\mathrm{CrV}$ alloys suppresses completely the magnetic order. For lower concentrations of $\mathrm{V}$, the magnetic phase boundary is continuously shifted toward the AF phase and finally vanishes with increasing $\mathrm{V}$ concentration. ${ }^{13,14}$ Therefore, intermixing at the interface or within very thin $\mathrm{Cr}$ layers during sample preparation can easily result in a nonmagnetic state $^{9}$ or in a state with pure AF ordering. ${ }^{10}$
Therefore, in order to test the intrinsic properties of the I-SDW state, it is important to control the intermixing and to use an alternative approach for tuning the system. Hydrogen adsorption on surfaces and interfaces with $\mathrm{Cr}$ provides an excellent possibility. Rotenberg et al. ${ }^{14}$ and Krupin et al. ${ }^{13}$ mapped the SDW phase diagram of $\operatorname{Cr}(110)$ and $\mathrm{Cr}_{100-\mathrm{x}} \mathrm{V}_{\mathrm{x}}(110)$ thin films grown on $\mathrm{W}(110)$ substrates by means of angle resolved photoemission as a function of the film composition, thickness and hydrogen surface coverage. Using a wedge-shaped thickness profile, they observed both AF and SDW phases separated by a nearly continues transition. Furthermore, they found that hydrogen adsorption on the outer surface of the film extends the region of the AF phase stability to thicker films. The AF stabilization was associated with an interplay between two-dimensional (2D) and three-dimensional Fermi surface nesting. ${ }^{13}$ In fact, Rotenberg et al. concluded that the Fermi contour for surface states plays an important role through an accidental symmetry upon hydrogen adsorption that favors the AF state.

To check this hypothesis. it is worth to investigate the modification of the SDW state under hydrogenation in $\mathrm{Cr} / \mathrm{V}$ superlattices. $\mathrm{V}$ exhibits an exothermal hydrogen solubility and can reversibly absorb hydrogen from the surrounding atmosphere. The influence of hydrogen loading on the magnetic properties has already been investigated for $\mathrm{Fe} / \mathrm{V}$ multilayers, where interlayer exchange coupling, Curie temperature of the Fe layers, and the Fe magnetic moments can be tuned with the hydrogen concentration in the $\mathrm{V}$ spacer layers. ${ }^{15,16}$ Our theory based on the model Hamiltonian approach taking into account the intermixing during the epitaxial growth ${ }^{17}$ has been proven to describe not only the main trends of $\mathrm{Fe} / \mathrm{V}$ upon hydrogen loading ${ }^{16,18}$ but also to predicted the possibility for a "remote control" of the Fe magnetic moment via hydrogen uptake in the V layer, which was confirmed experimentally by Remhof et al. ${ }^{19}$ The same theoretical approach, which does not use the nesting property of the $\mathrm{Cr}$ Fermi surface, was also used for the description of SDWs in thin Cr films. ${ }^{3}$ The aims of this paper are as follows.

(i) To review experimental studies that show the influence of hydrogen on SDW and AF states in $\mathrm{Cr} / \mathrm{V}(001)$ multilayers (Sec. II).

(ii) To develop the theoretical approach for calculating the magnetic structure in an external magnetic field which stimulates the transition from one magnetic state to another (Sec. III).

(iii) To explore if the proposed model can explain the observed transition from the SDW to the AF state under hydrogen loading and to discuss the driving forces of this transition (Sec. IV).

\section{MODIFICATION OF THE SDW STATE IN CR/V SUPERLATTICES VIA HYDROGEN UPTAKE IN V LAYERS}

For the investigation of the hydrogen influence on the SDW state, we consider a superlattice with thick $\mathrm{Cr}$ layers and relatively thin $\mathrm{V}$ layers. Hydrogen cannot penetrate into the $\mathrm{Cr}$ slabs and interaction with the SDW state can only 
occur via contact at the $\mathrm{H}-\mathrm{Cr}$ interface, similar to the adsorption of hydrogen on Cr surfaces as reported in Refs. 13 and 14 or more indirectly, via $d$-state hybridization of the $\mathrm{V}$ and $\mathrm{Cr}$ interface layers. The sample preparation and characterization of $\mathrm{Cr} / \mathrm{V}(001)$ superlattices was reported in detail by Kravtsov et al. ${ }^{9}$ In short, $\mathrm{Cr} / \mathrm{V}$ superlattices were grown by conventional dc sputtering methods in ultrahigh vacuum (UHV) onto polished $\mathrm{MgO}$ (001) substrate. The samples were covered by a thin catalytically active $\mathrm{Pd}$ layer to enhance hydrogen uptake and to prevent the $\mathrm{Cr} / \mathrm{V}$ heterostructures from corrosion. Structural characterization by $\mathrm{x}$-ray scattering revealed an excellent layer structure, a high degree of structural coherence, and a low interface roughness. However, interface intermixing at the monolayer scale cannot be excluded.

The SDW state of the superlattices was analyzed with unpolarized elastic neutron diffraction. Considering certain selection rules for magnetic neutron scattering, the propagation direction and the polarization (transverse or longitudinal) of the SDW can be determined. ${ }^{21}$ Furthermore, one can easily distinguish between a commensurate AF SDW state and an incommensurate SDW state. This is due to the fact that the bcc $\{100\}$ half-order peak is forbidden by the nuclear or atomic structure factors, but it is allowed for an antiferromagnetic $\mathrm{Cr}$ state, because of the doubling of the magnetic unit cell with respect to the chemical structure.

In these neutron scattering experiments, it was confirmed that the SDW state in $\mathrm{Cr} / \mathrm{V}$ superlattices is strongly affected by finite-size and proximity effects from neighboring $\mathrm{V}$ layers. ${ }^{9,20}$ Surprisingly, the $\mathrm{Cr}$ magnetism becomes completely suppressed in $\mathrm{Cr} / \mathrm{V}$ heterostructures at $\mathrm{Cr}$ layer thicknesses of less than $100 \AA$. The suppression is much stronger than previously reported by Almokhtar et al. ${ }^{8}$ and it is most likely due to some interface alloying. When increasing the $\mathrm{Cr}$ thickness beyond $100 \AA$, first, the $\mathrm{Cr}$ layers in $\mathrm{Cr} / \mathrm{V}$ show a transition to a commensurate SDW state and finally developed an incommensurate SDW for Cr layer thicknesses of more than $250 \AA$. Furthermore, in thick Cr layers, a stabilization of the longitudinal out-of-plane SDW was observed. This is in contrast to $\mathrm{Fe} / \mathrm{Cr}$ superlattices, where a transverse out-of-plane SDW is preferred. ${ }^{21}$ In $\mathrm{Cr}$ layers of $500 \AA$ thickness, the longitudinal SDW exists from below 50 up to $220 \mathrm{~K}$, between 220 and $250 \mathrm{~K}$, a commensurate AF phase occurs and coexists with the I-SDW phase, and above $250 \mathrm{~K}$ only the AF phase can be recognized up to $300 \mathrm{~K}$.

More recently, the $[\mathrm{Cr}(14 \AA) / \mathrm{V}(500 \AA)] \times 4$ superlattice was reinvestigated by thermal neutron scattering in order to analyze the effect that hydrogen loading has on the SDW state. ${ }^{24}$ By exposing the $\mathrm{Cr} / \mathrm{V}$ superlattice to a hydrogen atmosphere, hydrogen penetrates into the $\mathrm{V}$ layers while the $\mathrm{Cr}$ layers remain free of hydrogen. ${ }^{22,23}$ The hydrogen concentration in the V layers can thereby be controlled by the external hydrogen pressure.

The neutron scattering results from Ref. 24 are reproduced in Fig. 1. At a constant temperature of $220 \mathrm{~K}$ and in vacuum, we recognize the incommensurate SDW peaks at the reciprocal lattice position $\left(\begin{array}{ll}0 & 1 \pm \delta\end{array}\right), \delta=0.04$. No intensity is seen at the commensurate $\left(\begin{array}{lll}0 & 1 & 0\end{array}\right)$ position. Upon hydrogen loading, a commensurate peak develops at $\left(\begin{array}{lll}0 & 1 & 0\end{array}\right)$ on the expense of the incommensurate $\left(\begin{array}{ll}0 & 1 \pm \delta\end{array}\right)$ peaks for hy-

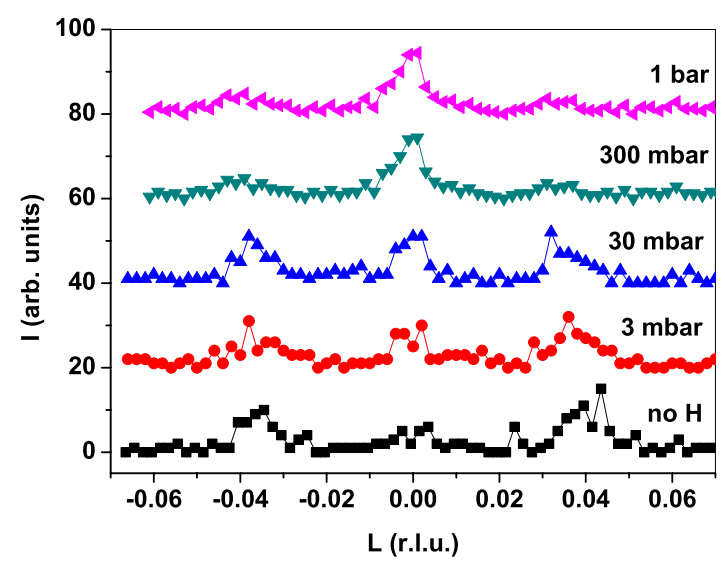

FIG. 1. (Color online) Neutron scattering scans taken in the $L$ direction through the $\mathrm{Cr}(010)$ position at temperature $T=220 \mathrm{~K}$. The satellite peaks observed at zero hydrogen pressure are at $\delta$ $\approx \pm 0.04$ r.l.u. and correspond to neutron scattering from the incommensurate SDW phase. The peak at $\delta=0$ is due to neutron scattering from the commensurate $\mathrm{AF}$ state. Data are reproduced from Ref. 24.

drogen pressures up to 300 mbar. At higher pressures, a saturation effect occurs and no further change in the position or in the intensity of the SDW peaks is observed. From these experiments, it is clear that hydrogen, solely residing in the $\mathrm{V}$ layers, can at least partially drive the SDW state from incommensurate to commensurate ordering. The position of the incommensurate SDW peaks change only slightly with hydrogen loading, and the main change is connected with a redistribution of intensity from incommensurate to commensurate SDW peaks. This means that, with hydrogen uptake, the volume fraction of the AF phase increases relative to the incommensurate SDW phase. At larger hydrogen pressure, the satellite reflections begin to shift toward the central reflection. Here, most likely, SDW-domains with different number of nodes coexist. ${ }^{5}$ The differences observed for the 300 mbar loading and for the 1000 mbar loading are rather small, suggesting that saturation is already achieved at 200 300 mbar.

The effect of temperature increase on the SDW state is very similar to the effect of hydrogen uptake. However, the temperature at which the SDW state is completely suppressed, indicated by the depression of the incommensurate ( $\left.\begin{array}{ll}0 & 1 \pm \delta\end{array}\right)$ peak intensity, is $10-20 \mathrm{~K}$ lower for the hydrogen loaded sample as compared to the pristine sample. Additionally, small volume fractions of the hydrogen loaded sample are in the AF state even at low temperatures $(20 \mathrm{~K})$. This has not been observed for the pristine sample. Obviously, the presence of hydrogen in the $\mathrm{V}$ layers favors the AF state within the adjacent $\mathrm{Cr}$ layers. This effect is completely reversible. The incommensurate SDW state is recovered by reducing the hydrogen pressure.

\section{THEORETICAL MODEL FOR CALCULATING THE HYDROGEN INFLUENCE ON THE SDW STATE IN CR/V MULTILAYERS}

As was established above, hydrogen loading leads to a transition from I-SDW to AF state in the $\mathrm{Cr}$ slabs of $\mathrm{Cr} / \mathrm{V}$ 
superlattices. Such behavior agrees with the observation reported in Ref. 13, that hydrogen adsorption on the outer surface of the $\mathrm{Cr}_{1-\mathrm{x}} \mathrm{V}_{\mathrm{x}}(110)$ alloy extends the regime of $\mathrm{AF}$ phase stability to thicker films in the wedge sample. Moreover, the AF phase was found to be very stable: it was detected at a vanadium concentration of up to $x \sim 0.08$ that is about twice as high as the concentration where in the bulk, the AF paramagnetic transition occurs. ${ }^{13}$ Therefore, we can conclude that the transition from SDW to AF state under hydrogen action is quite a general phenomenon. In both cases, hydrogen atoms do not penetrate into the $\mathrm{Cr}$ slab and hybridization of $\mathrm{Cr}-\mathrm{H}$ electronic states can have an impact only on interface $\mathrm{Cr}$ atoms. Each hydrogen atom absorbed in the $\mathrm{V}$ layers brings one electron into the system. However, these electrons are confined and the corresponding electronic states lay far below the Fermi level. ${ }^{25,26}$ Therefore, the influence of hydrogen cannot be explained via a change in the nesting property of the Cr Fermi surface. Even the Fermi surface of $\mathrm{V}$ will not be perturbed much by hydrogen. But some additional nesting of the interface $\mathrm{Cr}$ states is conceivable, stabilizing the AF state. However, suppression of the SDW state, which is observed for different surface orientations, can hardly be explained by the appearance of specific nesting vectors for the different directions. Therefore, an explanation of the hydrogen impact on the basis of electronic structure calculations is of large interest for a thorough understanding of the SDW in Cr.

We used a model Hamiltonian approach for itinerant electrons with real-space recursion methods for calculations of the magnetic structures. The model assumes the existence of two bands, one corresponding to the quasilocalized $d$ electrons and the other one to the itinerant $s p$ electrons. The Hamiltonian of the system reads

$$
\begin{aligned}
H= & \sum_{i, j, \alpha} E_{i j} \hat{d}_{i \alpha}^{+} \hat{d}_{i \alpha}+\sum_{\mathbf{k}, \alpha} E_{\mathbf{k}} \hat{c}_{\mathbf{k} \alpha}^{+} \hat{c}_{\mathbf{k} \alpha}+\sum_{\mathbf{k}, i, \alpha} V_{\mathbf{k} i} \hat{c}_{\mathbf{k} \alpha}^{+} \hat{d}_{i \alpha} \\
& +\sum_{i} U_{i} \hat{d}_{i \alpha}^{+} \hat{d}_{i \alpha} \hat{d}_{i-\alpha}^{+} \hat{d}_{i-\alpha}+\text { H.c. }
\end{aligned}
$$

Here, $\hat{d}_{i \alpha}^{+}\left(\hat{d}_{i \alpha}\right)$ and $\hat{c}_{\mathbf{k} \alpha}^{+}\left(c_{\mathbf{k} \alpha}\right)$ refer to the creation (annihilation) operators of $d$ electrons with spin $\alpha$ on atomic site $i$ and $s(p)$ electrons with quasimomentum $\boldsymbol{k}$ and spin $\alpha$, respectively. The energies of these electrons $E_{i i}$ and $E_{\mathbf{k}}$, hopping parameters $E_{i j}$, and $s p$ - $d$ hybridization potential $V_{\mathbf{k} i}$ are taken to be spin independent. Mean field approximation is used for treating the Coulomb repulsion on site $U_{i}$. form

Equations for $d$-electron Green's functions $G_{i j}^{\alpha}$ have the

$$
\left(\omega-E_{i}^{\alpha}\right) G_{i j}^{\alpha}(\omega)-\sum_{l} V_{i l} G_{l j}^{\alpha}(\omega)=\delta_{i j}
$$

where the effective $d$-electron energy $E_{i}^{\alpha}$ and hopping integrals $V_{i l}$ contain contributions associated with $s p$ - $d$ hybridization

$$
E_{i}^{\alpha}=E_{i i}+\sum_{\mathbf{k}} \frac{V_{i \mathbf{k}} V_{\mathbf{k} i}}{\omega-E_{\mathbf{k}}}+U_{i} N_{i}^{-\alpha}
$$

$$
V_{i l}=E_{i l}+\sum_{\mathbf{k}} \frac{V_{i \mathbf{k}} V_{\mathbf{k} l}}{\omega-E_{\mathbf{k}}} .
$$

Thus, $E_{i}^{\alpha}$ includes an imaginary part, which is determined by the $s p-d$ interaction

$$
\Gamma_{i}=\operatorname{Im} \sum_{\mathbf{k}} \frac{V_{i \mathbf{k}} V_{\mathbf{k} i}}{\omega-E_{\mathbf{k}}} .
$$

In what follows, all energy parameters are measured in $\Gamma$ units, which is assumed to be independent on site $i$.

Self-consistency condition (3) connects the energy of $d$ electrons $\left(E_{i}^{\alpha}\right)$ with occupation number $\left(N_{i}^{-\alpha}\right)$, which can be calculated via the imaginary part of the Green's function $\left(G_{i i}^{-\alpha}\right)$. Equations (2) are solved by real-space recursion methods, taking into account, for simplicity, hopping Eq. (4) inside the first-nearest neighbor shell of each atom. Poles of the Green's function can be found numerically and than for occupation numbers analytical expressions can be obtained. This procedure increases effectively the accuracy and the convergence rate of the self-consistent calculations.

The outlined theory contains only very few phenomenological dimensionless parameters, which are universal for each of the elements. These parameters determine the position of the $d$ states relative to the Fermi level $\left[\left(E_{0 i}-\varepsilon_{\mathrm{F}}\right) / \Gamma\right]$, the on-site Coulomb repulsion $(U / \Gamma)$, and the hopping integrals between nearest neighbors $(V / \Gamma)$. The parameters for $\mathrm{Cr}$ and $\mathrm{V}$ were chosen in accordance with data from previous calculations for $\mathrm{Cr},{ }^{3} \mathrm{Fe} / \mathrm{V},{ }^{16,18,19}$ and $\mathrm{Fe} / \mathrm{Cr}$ (Ref. 17) systems.

Our previous calculations of the effect of hydrogen on the magnetic properties of $\mathrm{Fe} / \mathrm{V}$ multilayers ${ }^{16,19}$ have shown that interface intermixing has to be taken into account for an adequate description of experimental data. Therefore, the growth related intermixing should be included in the theoretical modeling of SDW-state transformation. For the description of intermixing, we put forward an algorithm, which assumes that a fraction of the deposited atoms is exchanged with atoms of the growing film. As a result, adatoms are floating on the upper layers. The only parameter of the algorithm is the ratio of atoms that are exchanged with the substrate atoms during the deposition of the next layer, which is always smaller than unity $(\zeta<1)$. Due to the existence of a preferred direction (the growth direction), the chemical profile near the interface becomes asymmetric. Using the floating algorithm for modeling the interface mixing in superlattices, combined with self-consistent calculations of atomic magnetic moments, we could explain (see Ref. 17) the magnetic asymmetry of interfaces reported for $\mathrm{Cr} / \mathrm{V}$ heterostructures by Almokhtar et al. ${ }^{8}$

Hydrogen absorption inside $\mathrm{V}$ layers was modeled by using a procedure that fills randomly chosen octahedral interstitial sites. In the $\mathrm{V} / \mathrm{Cr}$ system with intermixing, only those interstitial sites are available for hydrogen occupation that have only $\mathrm{V}$ atoms among the nearest neighbor or second neighbor lattice sites. This procedure automatically hinders any hydrogen atom to occupy sites in the $\mathrm{Cr}$ slabs and generates a concentration gradient at the interface. ${ }^{17,19}$ 
Self-consistent calculations of the magnetic structure were performed for the generated distribution of $\mathrm{V}, \mathrm{Cr}$, and $\mathrm{H}$ atoms in the bcc lattice.

The most challenging problem is to determine the number of nonequivalent atoms in a unit cell, for which the calculations of magnetic moments have to be performed selfconsistently. The total number of atomic layers in one period of the experimentally studied Cr/V sample is about 360 (350 $\mathrm{Cr}$ layers and $10 \mathrm{~V}$ layers). For modeling of intermixing, we used a $8 \times 8$ in-plane unit cell with periodic boundary conditions. Thus, the total number of nonequivalent atoms exceeded 23 000. Calculations with absorbed hydrogen were even more complex. Therefore, using an analytical representation for the electronic $d$ state occupation numbers proved to be extremely important.

In a system with tens of thousands of nonequivalent atoms, one might expect a large number of different selfconsistent solutions with a close energy distribution. To separate the solutions that correspond to the SDW state, we performed our calculations under the assumption of an external magnetic field $H$ that stimulates the transition to the SDW state. ${ }^{3}$ The introduction of an external magnetic field into the Hamiltonian (1), within the collinear framework, leads to a shift in the bands for electrons with different spin projections by the value $h=\mu_{\mathrm{B}} H / \Gamma$ (in $\Gamma$ units). In order to obtain the SDW structure with a period $\Lambda$, the periodic or staggered field was chosen to mimic the distribution of the magnetic moments in the SDW,

$$
h_{i}=(-1)^{i+1} h^{*} \sin \frac{2 \pi}{\Lambda}(i-1), \quad i=1,2 \ldots \Lambda,
$$

with amplitude $h^{*}=10^{-3}$. After convergence, the field was switched off and self-consistent calculations were continued with initial states corresponding to the self-consistent solution in the external field (6).

\section{HYDROGEN AS TRIGGER FOR TRANSITION FROM SDW TO AF STATE IN CR/V STRUCTURES}

Calculations of the electronic structure were performed for $\mathrm{Cr}_{350} / \mathrm{V}_{10}$ (the subscripts indicate the number of atomic layers) with in-plane periodicity $8 \times 8$ atoms. Interface alloying was introduced into the system using a floating algorithm with intermixing parameter $\zeta$ as described above. First, selfconsistent calculations of magnetic moments were performed in a periodic external field (6). Then, the field was switched off and the calculations were repeated without field. In all cases, independent of the intermixing parameter $\zeta$, the final self-consistent solution was found to be an incommensurate SDW state. However, due to the random algorithm in the intermixing, modeling the profile of the magnetization can be slightly different even for the same value of $\zeta$. In Figs. 2(a) and 3(a), the average magnetic moments per layer are depicted for $\zeta=0.25$. Layers $1-10$ refer to the vanadium layers, whereas all other layers refer to chromium. Layers 60-320 are not shown for clarity of the figure. Small variations in the magnetic profiles can be seen near the $\mathrm{Cr} / \mathrm{V}$ interfaces in Figs. 2(a) and 3(a). For other intermixing parameters $(\zeta)$, the
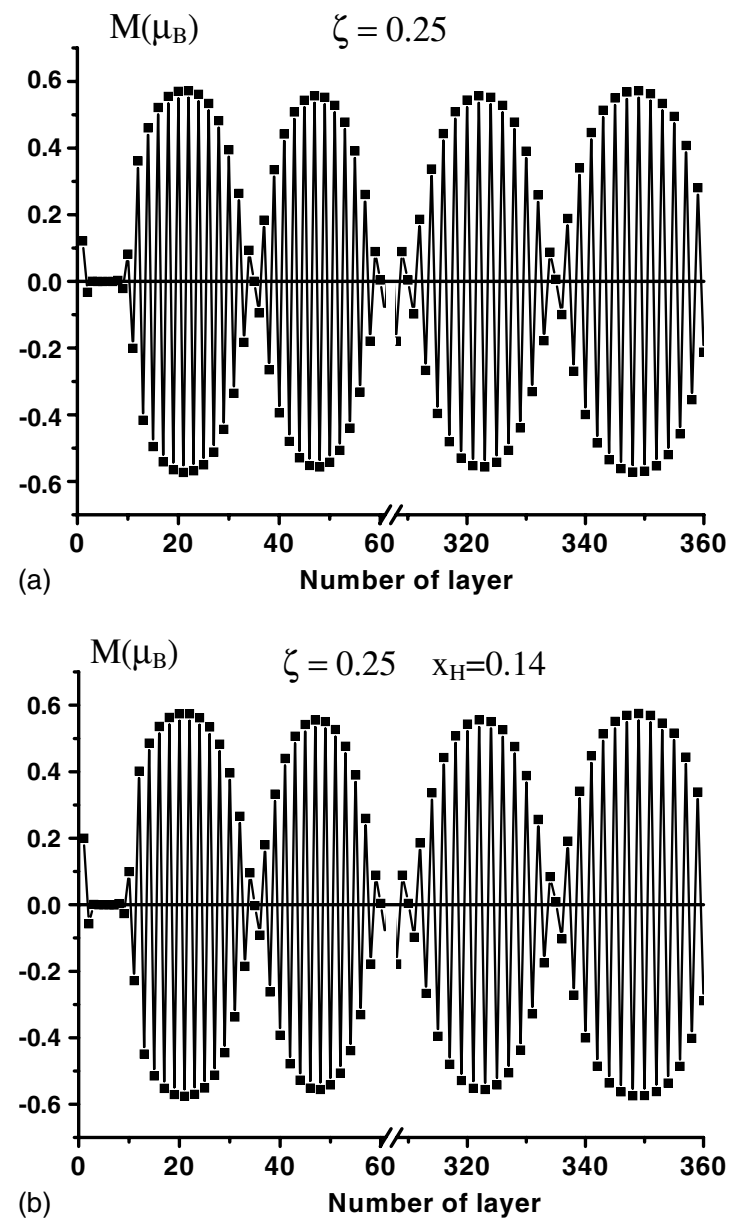

FIG. 2. Magnetic profile of a $\mathrm{V}_{10} / \mathrm{Cr}_{350}$ superlattice before (a) and after hydrogen uptake in $\mathrm{V}$ layers (b). Chemical intermixing at the $\mathrm{Cr} / \mathrm{V}$ interface is modeled with the intermixing parameter $\zeta$ $=0.25$ and the hydrogen concentration in the V layers is $x_{H}=0.14$.

variations are larger, but they are always located within the interface region.

When convergence for the hydrogen free structure was achieved, the hydrogen atoms were inserted into the system and again self-consistent calculations were performed starting from the initial SDW-state obtained without hydrogen. The atomic positions of $\mathrm{V}$ and $\mathrm{Cr}$ atoms were taken to be exactly the same as in the preceding calculations without $\mathrm{H}$. The stoichiometry was not fixed and the hydrogen concentration $x_{H}=\mathrm{H} / \mathrm{V}$ atoms was considered as a continuous parameter, which can be adjusted via the applied hydrogen pressure at a given temperature. The results of our calculations clearly show that hydrogen acts as trigger for the transition from SDW to AF state. Figure 2(b) shows the magnetic profile of the system with a hydrogen concentration of $x_{H}=0.14$. The SDW state in $\mathrm{Cr}$ is still present but the values for the $\mathrm{Cr}$ magnetic moments, as well as the induced moments on the $\mathrm{V}$ atoms, have increased in the interface region as compared to the virgin structure [Fig. 2(a)]. This result resemble the results obtained on $\mathrm{Fe} / \mathrm{V}$ heterostructures, where absorption of hydrogen in $\mathrm{V}$ layers leads to an increase in the Fe magnetic moments. ${ }^{16,19}$ When the amount of 

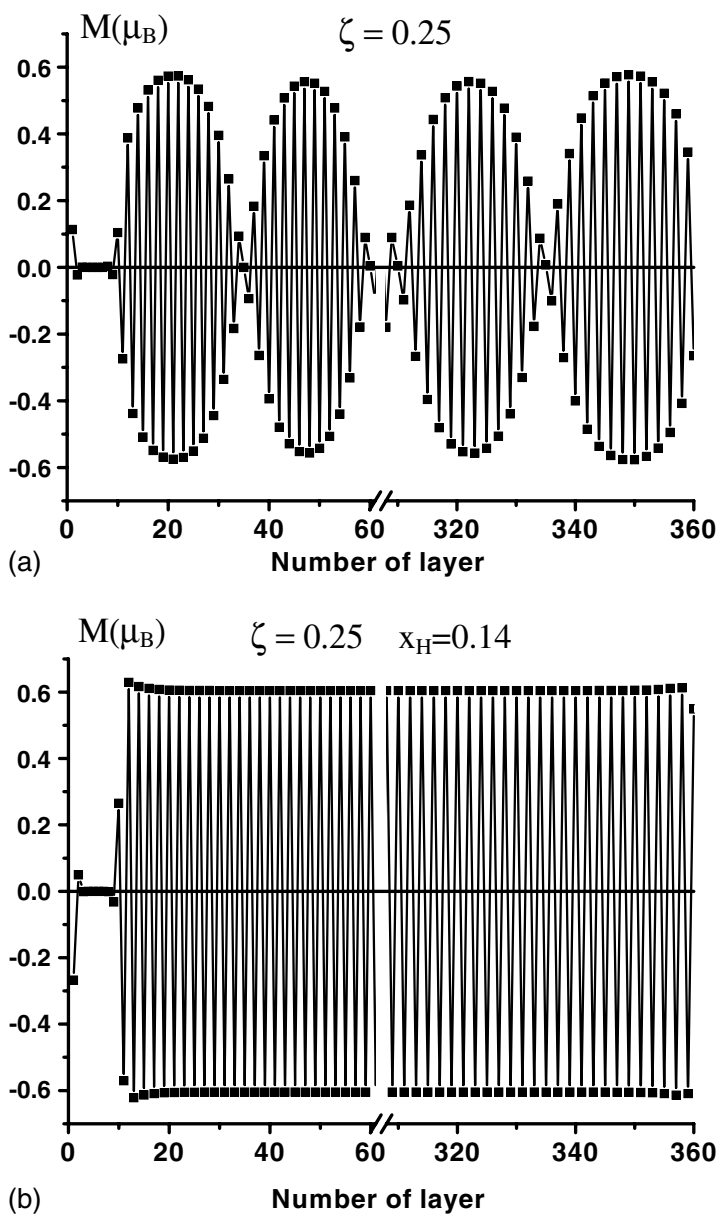

FIG. 3. Magnetic profile of a $\mathrm{V}_{10} / \mathrm{Cr}_{350}$ superlattice before (a) and after hydrogen uptake in $\mathrm{V}$ layers (b). Chemical intermixing at the $\mathrm{Cr} / \mathrm{V}$ interface is modeled with the intermixing parameter $\zeta$ $=0.25$ and the hydrogen concentration in the $\mathrm{V}$ layers is $x_{H}=0.14$

hydrogen in the $\mathrm{V}$ spacer layers increases, a sudden transition from the SDW state to AF ordering takes place as can be seen in Fig. 3(b) at $x_{H}=0.5$. The mechanism of the transition is not connected with specific nesting properties at the Fermi surface for $\mathrm{Cr}$ atoms near the $\mathrm{V} / \mathrm{Cr}$ interface, ${ }^{11}$ but with a reduction in the threshold that separates the metastable SDW and the $\mathrm{AF}$ ground states.

Evolution of threshold with intermixing and with hydrogen loading can be studied via calculations of hysteresis loops in a local magnetic field (6) as it was described in Ref. 3 for bulk Cr. There, we have shown that the smaller the distance between nodes (wavelength of SDW), the smaller the field that is required to enable a transition from I-SDW to AF. The influence of hydrogen leads to the same effect as a decrease in the SDW wavelength $\Lambda$. From this, we infer that the threshold between I-SDW and AF states becomes lower with hydrogen loading.

For other intermixing parameters, we obtain similar scenarios for the SDW-AF transition. In some cases, hydrogen annihilates not all SDW nodes, but only a few of them, and in this case, the influence of hydrogen is similar to the influence that a temperature increase has on the number of nodes in $\mathrm{Cr}$ slabs, for instance, in $\mathrm{Cr} / \mathrm{Cr}_{97,5} \mathrm{Mn}_{2,5}(001)$ structures. ${ }^{5}$
In our model, the stability of the SDW state depends on the intermixing parameter in a nontrivial way. For $\zeta \ll 1$, the $\mathrm{Cr} / \mathrm{V}$ and $\mathrm{V} / \mathrm{Cr}$ interfaces are almost symmetric and very narrow. With increasing intermixing parameter, a small fraction of $\mathrm{V}$ atoms penetrate farther from the interface. In the limiting case $\zeta \approx 1$, almost all $\mathrm{V}$ atoms flow up from interface and the interface will be ideally flat without intermixing. Note also that the suggested mechanism of intermixing during growth promotes floating of only one monolayer of $\mathrm{V}$ into the $\mathrm{Cr}$ slab. Therefore, the concentration of $\mathrm{V}$ in the $\mathrm{Cr}$ layers is always very small. In real samples, the intermixing could depend on the lateral coordinates and could be considerably larger than assumed in our model. Furthermore, real samples should be considered as composed of a set of systems with different $\zeta$ values. Calculations have shown that for a fixed value of $\zeta$, the SDW nodes can only disappear with increasing hydrogen concentration $x_{H}$, in other words, adding hydrogen to a system with a fixed value of $\zeta$ will decrease the number of nodes.

Our calculations can explain all the main features of the magnetic structure of $\mathrm{Cr} / \mathrm{V}$ multilayers and their behavior upon hydrogen uptake. For moderate hydrogen pressures, there are parts of the interface where intermixing and local hydrogen concentration in the $\mathrm{V}$ layers trigger the transitions from SDW to AF as it is shown in Fig. 3. These parts will change the intensity ratio between SDW and AF peaks in neutron spectra. The transition to the ground state occurs when all nodes are successively being annihilated. The annihilation process changes the effective wavelength of the SDW, thus shifting the SDW peaks toward the central AF peak. Simultaneously, the amplitude of the SDW decreases to zero. This is the transition that is actually observed in experiments when $\mathrm{Cr}$ is in contact with hydrogen.

\section{CONCLUSIONS}

We have presented experimental and theoretical studies of the SDW stability in $\mathrm{V} / \mathrm{Cr}(001)$ superlattices with thick $\mathrm{Cr}$ layers. Neutron scattering experiments have shown that hydrogen uptake in thin $\mathrm{V}$ spacer layers favors a transition from the incommensurate SDW state to the commensurate antiferromagnetic state. These results, together with recent observations of a change in the magnetic phase diagram in $\mathrm{Cr}_{1-\mathrm{x}} \mathrm{V}_{\mathrm{x}}(110)$ alloy films under the action of $\mathrm{H}$ adsorption on the (110) surface, ${ }^{11,13}$ confirm that the destabilization of the incommensurate SDW phase and the stabilization of the commensurate $\mathrm{AF}$ phase is general phenomenon. It can hardly be explained by the appearance of an additional 2D nesting effect at $\mathrm{Cr}$ surface or interface, or at surface layers under the action of hydrogen.

Theoretical calculations based on a model Hamiltonian approach for itinerant electrons provide a natural explanation for the observed phenomena which do not rely on specific nesting properties of the $\mathrm{Cr}$ or V Fermi surfaces. In pristine $\mathrm{Cr}$, the incommensurate SDW state is quite stable, although all recent $a b$ initio theories have shown that it is not the ground state of the system. ${ }^{1,2}$ The stability is due to the energy barrier, which separates the incommensurate SDW state from the more stable AF ground state. The height of barrier 
for the annihilation of the SDW nodes was found to be higher than for their shift. As results magnetic nodes cannot be easily destroyed. They only shift under the action of external perturbations. Hydrogen reduces this barrier for annihilation of the nodes and therefore, it works as a trigger for the SDW-AF transition. The presented results support the insight into the SDW formation without reference to the nesting property of the Fermi surface. ${ }^{3}$

\section{ACKNOWLEDGMENTS}

We would like to thank E. Kravtsov for permitting us to reproduce Fig. 1 from his work. This work was partially supported by DFG-RFBR cooperative under Grant No. 1002-91330, by RFBR under Projects No. 07-02-01065, No. AVCP 2.1.1/4215, No. SFB 491 Bochum/Duisburg, and by Nordic Center of Excellence on Hydrogen Storage Materials.
${ }^{1}$ R. Hafner, D. Spišák, R. Lorenz, and J. Hafner, Phys. Rev. B 65, 184432 (2002).

${ }^{2}$ S. Cottenier, B. De Vries, J. Meersschaut, and M. Rots, J. Phys.: Condens. Matter 14, 3275 (2002).

${ }^{3}$ V. M. Uzdin and C. Demangeat, J. Phys.: Condens. Matter 18, 2717 (2006)

${ }^{4}$ D. Aernout, C. L'abbé, M. Rots, H. Fritzsche, and J. Meersschaut, Phys. Rev. B 73, 134419 (2006).

${ }^{5}$ E. E. Fullerton, J. L. Robertson, A. R. E. Prinsloo, H. L. Alberts, and S. D. Bader, Phys. Rev. Lett. 91, 237201 (2003).

${ }^{6}$ H. Zabel, J. Phys.: Condens. Matter 11, 9303 (1999).

${ }^{7}$ K. Mibu and T. Shinjo, J. Phys. D 35, 2359 (2002).

${ }^{8}$ M. Almokhtar, K. Mibu, A. Nakanishi, T. Kobayashi, and T. Shinjo, J. Phys.: Condens. Matter 12, 9247 (2000).

${ }^{9}$ E. Kravtsov, R. Brucas, B. Hjörvarsson, A. Hoser, A. Liebig, G. J. McIntyre, M. A. Milyaev, A. Nefedov, L. Paolasini, F. Radu, A. Remhof, V. V. Ustinov, F. Yakhou, and H. Zabel, Phys. Rev. B 76, 024421 (2007).

${ }^{10}$ H. Fritzsche, S. Bonn, J. Hauschild, K. Prokes, and J. Klenke, Eur. Phys. J. B 36, 175 (2003).

${ }^{11}$ E. Rotenberg, B. K. Freelon, H. Koh, A. Bostwick, K. Rossnagel, A. Schmid, and S. D. Kevan, New J. Phys. 7, 114 (2005).

${ }^{12}$ N. Jiko, K. Mibu, and M. Takeda, Phys. Rev. B 71, 014414 (2005).

${ }^{13}$ O. Krupin, E. Rotenberg, and S. D. Kevan, Phys. Rev. Lett. 99, 147208 (2007).

${ }^{14}$ E. Rotenberg, O. Krupin, and S. D. Kevan, New J. Phys. 10, 023003 (2008).
${ }^{15}$ V. Leiner, K. Westerholt, A. M. Blixt, H. Zabel, and B. Hjörvarsson, Phys. Rev. Lett. 91, 037202 (2003).

${ }^{16}$ V. Uzdin, K. Westerholt, H. Zabel, and B. Hjörvarsson, Phys. Rev. B 68, 214407 (2003).

${ }^{17}$ V. M. Uzdin and W. Keune, J. Phys.: Condens. Matter 19, 136201 (2007).

${ }^{18}$ V. M. Uzdin and L. Häggström, Phys. Rev. B 72, 024407 (2005).

${ }^{19}$ A. Remhof, G. Nowak, H. Zabel, M. Björck, M. Pärnaste, B. Hjörvarsson, and V. Uzdin, EPL 79, 37003 (2007).

${ }^{20}$ E. Kravtsov, A. Nefedov, F. Radu, A. Remhof, H. Zabel, R. Brucas, B. Hjörvarsson, A. Hoser, and G. J. McIntyre, J. Phys.: Condens. Matter 17, 3143 (2005).

${ }^{21}$ P. Bödeker, A. Schreyer, and H. Zabel, Phys. Rev. B 59, 9408 (1999).

${ }^{22}$ B. Hjörvarsson, A. Stierle, G. Song, and H. Zabel, Vacuum 52, 291 (1999).

${ }^{23}$ V. Leiner, H. Zabel, J. Birch, and B. Hjörvarsson, Phys. Rev. B 66, 235413 (2002).

${ }^{24}$ E. Kravtsov, A. Nefedov, G. Nowak, K. Zhernenkov, H. Zabel, B. Hjörvarsson, A. Liebig, A. Hoser, G. J. McIntyre, L. Paolasini, and A. Remhof, J. Phys.: Condens. Matter 21, 336004 (2009).

${ }^{25}$ P. H. Andersson, L. Fast, L. Nordström, B. Johansson, and O. Eriksson, Phys. Rev. B 58, 5230 (1998).

${ }^{26}$ S. Ostanin, V. M. Uzdin, C. Demangeat, J. M. Wills, M. Alouani, and H. Dreysse, Phys. Rev. B 61, 4870 (2000). 